The objective of this study was to examine whether 10-year risk for incident stroke is associated with cognitive decline.

Methods Study sample comprised of 4512 men and 1741 women, mean age 55.6 years, from the Whitehall II study, a longitudinal British cohort study. The Framingham Stroke Risk Profile was used to assess 10-year risk of stroke. It incorporates age, systolic blood pressure, diabetes mellitus, smoking status, prior cardiovascular disease, atrial fibrillation, left ventricular hypertrophy, and use of hypertensive medication. Measures of cognitive function consisted of tests of reasoning, memory, phonemic and semantic fluency, and vocabulary, assessed three times over 10 years. Linear mixed models were used to determine longitudinal associations between stroke risk and subsequent cognitive decline over 10 years.

Results Higher stroke risk at baseline was associated with faster rate of cognitive in tests of reasoning, verbal fluency, vocabulary and global cognition. For example, compared to persons in the low stroke risk group $(<2.5 \%)$, those in the moderate stroke risk group $(2.5 \leq$ stroke risk $<5 \%)$ and the high stroke risk group $(\geq 5 \%)$ had a $12.5 \%$ and $43.8 \%$ faster rate of decline in, phonemic fluency respectively.

Conclusions Higher 10-year stroke risk in middle age is associated with faster rate of cognitive decline in more than one cognitive domain. These results support early targeting of vascular risk factors to prevent or delay cognitive decline.

\section{1-4.5 PROGNOSTIC VALUE OF A NOVEL CLASSIFICATION SCHEME OF CLINICAL SYMPTOMS AND SIGNS OF HEART FAILURE ADJUSTED FOR MAJOR CONFOUNDERS}

doi:10.1136/jech.2011.142976a.34

\begin{abstract}
1,2M Severo, ${ }^{*}{ }^{1,3} \mathrm{R}$ Gaio, ${ }^{4} \mathrm{~J}$ Pimenta, ${ }^{4} \mathrm{C}$ Paulo, ${ }^{4} \mathrm{M}$ Patacho, ${ }^{4} \mathrm{P}$ Bettencourt, ${ }^{1,2} \mathrm{~A}$ Azevedo. ${ }^{1}$ Department of Hygiene and Epidemiology, University of Porto Medical School, Porto, Portugal; ' ${ }^{2}$ Institute of Public Health of the University of Porto, Porto, Portugal; ${ }^{3}$ Department of Mathematics, University of Porto Science School, Porto, Portugal; ${ }^{4}$ Heart Failure Clinic, Department of Internal Medicine, Hospital S. João, Porto, Portugal
\end{abstract}

Introduction Only one third of patients suspected of having heart failure (HF) see this diagnosis confirmed. Gender, age, education and obesity are major determinants of unconfirmed suspicions.

Objective To assess the impact of including major confounders in classification schemes based on clinical data solely (model 1-11 symptoms/signs) or considering objective evidence of cardiac dysfunction (model 2).

Methods Cross-sectional evaluation of 1115 community participants aged $\geq 45$ years, 2006-2008. The individuals were classified by Latent Class Analysis with concomitant variables. The classification's prognostic value was assessed by the association with 6 year mortality in an independent sample of 753 subjects.

Results Bayesian Information criteria suggested the best solutions for model 1 and 2 was 2- and 3-class, respectively; the best solution for both models considering concomitant variables was 3-class.

Class 1 had high endorsement probabilities for all items (symptomatic HF); class 2 had high probability for volume overload and objective evidence of cardiac dysfunction and lower probability for subjective troubled breathing (asymptomatic cardiac dysfunction); class 3 had low endorsement probabilities for all items (non-cases).

The sex- and age-adjusted 6-year absolute risk of death was $13.5 \%, 4.3 \%$ and $2.7 \%$ for class 1,2 and 3 , respectively, in model 1 ; for model 2 it was $10.2 \%, 4.2 \%$ and $3.2 \%$, respectively.

Conclusions When relying only on clinical data and not considering confounders, we were only able to distinguish symptomatic HF from the normal population. Considering confounders and evidence of cardiac dysfunction improved the discriminative power to distinguish a third group with asymptomatic cardiac abnormalities.

\section{1-4.6 ALCOHOL-INDUCED DAMAGE TO HEART MUSCLE RATHER THAN ATHEROSCLEROSIS MAY DRIVE THE ASSOCIATION OF CIRCULATORY DISEASE WITH HAZARDOUS DRINKING IN RUSSIA}

doi:10.1136/jech.2011.142976a.35

${ }^{1} \mathrm{D}$ Leon, ${ }^{*}{ }^{2} \mathrm{~S}$ Borinskaya, ${ }^{1} \mathrm{~A}$ Gil, ${ }^{3} \mathrm{~N}$ Kiryanov, ${ }^{1} \mathrm{M}$ McKee, ${ }^{4} \mathrm{~A}$ Oralov, ${ }^{4} \mathrm{~L}$ Saburova, ${ }^{1} \mathrm{O}$ Savenko, ${ }^{5} \mathrm{~V}$ Shkolnikov, ${ }^{3} \mathrm{M}$ Vasilev, ${ }^{6} \mathrm{H}$ Watkins. ${ }^{1}$ London School of Hygiene 8 Tropical Medicine, London, UK; ${ }^{2}$ Vavilov Institute of General Genetics, Moscow, Russia; ${ }^{3}$ Izhevsk State Medical Academy, Izhevsk, Russia; ${ }^{4}$ Social Technologies Institute, Izhevsk, Russia; ${ }^{5}$ Max Planck Institute for Demographic Research, Rostock, Germany; ${ }^{6}$ Department of Cardiovascular Medicine, University of Oxford, Oxford, UK

Background Circulatory disease mortality in Russia is associated with hazardous drinking. Over the past 40 years there have been major fluctuations in mortality from circulatory disease that are closely correlated with deaths from acute alcohol poisoning $(\mathrm{r}=0.8$ among working-age men). In a case-control study (2003-2005) hazardous drinking was associated with deaths attributed to ischaemic heart disease. However, rather than hazardous alcohol consumption increasing risk of atherosclerotic disease and subsequent myocardial infarction, these effects could be due to misclassified non-atherosclerotic damage to the heart induced by heavy drinking as occurs in extreme form in alcoholic cardiomyopathy.

Methods A population-based sample of 1052 men aged 30-60 years living in Izhevsk (a medium-sized Russian city) were examined (2008-2009). Information about drinking was obtained by interview of proxy informants (mainly spouses or partners). Levels of Btype natriuretic peptide (BNP - a sensitive and highly specific marker of heart muscle stress) and an atherogenic index (ApoB to ApoA1 ratio) were measured in blood.

Results Compared to abstainers, men who drank hazardously had an increased risk of being in the top 20\% of BNP (OR 4.80, 95\% CI 2.29 to 10.1) adjusted for age and BMI, with non-hazardous drinkers being intermediate in risk. The equivalent association for the ApoB/ ApoA1 ratio was in the opposite direction (OR 0.31, 0.16 to 0.61 ). Conclusion Hazardous drinkers show a lipoprotein profile associated with a reduced risk of atherosclerotic disease. However, they have raised levels of BNP. Taken together these results support the hypothesis that hazardous drinking among Russian men may induce non-atherosclerotic heart muscle damage.

\subsection{NUTRITION}

\section{Chair: Prof. K. Srinath Reddy, India 01-5.1 CLUSTER-RANDOMISED CONTROLLED TRIAL OF AN EARLY CHILDHOOD OBESITY PREVENTION PROGRAM: THE MELBOURNE INFANT FEEDING, ACTIVITY AND NUTRITION TRIAL (InFANT) PROGRAM}

doi:10.1136/jech.2011.142976a.36

${ }^{1} \mathrm{~K}$ Hesketh, ${ }^{*}{ }^{1} \mathrm{~K}$ Campbell, ${ }^{1} \mathrm{D}$ Crawford, ${ }^{1} \mathrm{~J}$ Salmon, ${ }^{1} \mathrm{~K}$ Ball, ${ }^{1} \mathrm{~S}$ McNaughton, ${ }^{2} \mathrm{Z}$ McCallum. ${ }^{1}$ Centre for Physical Activity and Nutrition Research, Deakin University, Melbourne, Victoria, Australia; ${ }^{2}$ University of Melbourne, Melbourne, Victoria, Australia

Introduction This study aimed to assess the effectiveness of a childfocused early obesity prevention intervention for first-time parents in existing social networks.

Methods The Melbourne InFANT Program is a cluster-randomised controlled trial involving 542 families from 62 first-time parent groups in Melbourne, Australia (87\% recruitment; $90 \%$ retention). It focuses on positive diet, physical activity and reduced sedentary behaviours from 3 to 18 months of age. 
Results At intervention conclusion, children's objectively measured physical activity was similar across groups. Maternal beliefs about television and diet and corresponding child behaviours were more favourable in intervention than control participants. For example, fewer intervention group mothers believed television is helpful for development (67\% vs $43 \%$ ), and parents should offer other foods if their child doesn't eat their meal ( $44 \%$ vs $20 \%$; both $\mathrm{p}<0.001)$. More intervention mothers believed parents should include fruit or vegetables in all children's meals and snacks ( $96 \%$ vs $91 \%, p=0.04)$, and that television should be turned off when children are eating $(90 \%$ vs $81 \%, p=0.02$ ). Compared to control group infants, those in the intervention group were significantly more likely to consume two or more serves of fruit ( $81 \%$ vs $70 \%, p=0.03$ ), vegetables $(56 \%$ vs $42 \%$, $\mathrm{p}=0.04)$ and both fruit and vegetables daily (39\% vs $26 \%, \mathrm{p}<0.001)$ and watched an average of $92 \mathrm{~min}$ less television per week ( $\beta=-13.8,95 \%$ CI -26.4 to -1.2$)$.

Conclusions The Melbourne InFANT Program, a low-dose, low-cost obesity prevention intervention, shows promising impact on parent attitudes and beliefs and on children's diet and television viewing behaviours but not physical activity.

\section{$01-5.2$ MEAT, FISH AND ESOPHAGEAL CANCER RISK: A SYSTEMATIC REVIEW AND META-ANALYSIS}

doi:10.1136/jech.2011.142976a.37

${ }^{1} \mathrm{M}$ Salehi, ${ }^{* 2} \mathrm{~F}$ Kolahdooz, ${ }^{1} \mathrm{M}$ Moradi, ${ }^{3} \mathrm{M}$ H Salehi, ${ }^{1} \mathrm{M}$ Nojomi. ${ }^{1}$ Tehran University of Medical Sciences, Tehran, Iran; ${ }^{2}$ Food \& Drug Division, Ministry of Health and Medical Education, Tehran, Iran; ${ }^{3}$ Mashhad University of Medical Sciences, Mashhad, Iran

Introduction Esophageal cancer is the 8th most common cancer and the 6 th leading cause of cancer mortality worldwide. Mortality from this cancer is high and despite improvements in treatment, the 5 year survival rate still remains low at around $10-13 \%$. The associations between some of risk factors with esophageal cancer are well defined while the role of diet remains controversial. Thus, we sought to examine quantitatively the association between intake of meat and fish and esophageal cancer.

Methods We searched major database for published studies (January 1990 through January 2011) on the associations between consumption of total meat, red meat, processed meat, poultry, and fish and risk of esophageal cancer. Random-effects models were used to pool study results. We conducted subgroup analyses by, histologic subtypes of esophageal cancer, nationality, and potential confounders.

Results We identified three cohort studies and 30 case control studies. The pooled RR of esophageal cancer for intakes of the highest group compared with the lowest group of total meat, red meat, processed meat, poultry and fish were 1.02 (95\% CI 0.89 to 1.18), 1.42 (95\% CI 1.09 to 1.86 ), 1.41 (95\% CI 1.12 to -1.78$), 0.87$ ( $95 \%$ CI 0.60 to 1.24 ), 0.77 (95\% CI 0.62 to 0.97 ) respectively.

Conclusion Our results suggest that high intake of red meat and processed meat may be associated with a higher risk of esophageal cancer, whereas high fish intake may be related to a lower risk.

\section{1-5.3 THE ROLE OF MILK AND DAIRY PRODUCTS IN CHILDHOOD OBESITY: EVIDENCE FROM THE HONG KONG'S “CHILDREN OF 1997" BIRTH COHORT}

doi:10.1136/jech.2011.142976a.38

L L Shi, ${ }^{*}$ M Tarrant, L L Hui, M K Kwok, T H Lam, G M Leung, C M Schooling. The University of Hong Kong, Hong Kong, China

Introduction Observational studies, mainly from western populations, suggest that dairy product consumption is inversely associated with adiposity. However, in such populations, there is a limited range of dairy product intake while dietary intake and obesity share social patterning making evidence from non-western developed settings valuable in distinguishing whether the observed associations are biologically mediated or socially confounded.

Methods We used multivariable linear regression to examine the adjusted association of the frequency of milk or other dairy product consumption at 11 years with clinically measured body mass index (BMI) $z$-scores at about 13 years, relative to the 2007 WHO growth reference in a large $(n=8327)$, population-representative Chinese birth cohort, comprising 88\% of all births in Hong Kong in April and May 1997.

Results Of the original 8327 cohort members, 7933 are alive, participating and living in Hong Kong. At approximately 13 years, 7488 had clinically assessed BMI. Socio-economic position was positively associated with frequency of milk and other dairy product consumption. Neither milk nor other dairy product consumption was associated with BMI z-score (milk $-0.02,95 \% \mathrm{CI}-0.06$ to 0.03 and dairy products $0.03,95 \% \mathrm{CI}-0.01$ to 0.07 ), adjusted for sex, mother's birthplace, highest parental education, pubertal stage, physical activity and other food consumption.

Conclusions In a non-western setting, milk and other dairy product consumption was not associated with adiposity, suggesting that any observed anti-obesigenic effects in western settings may be due to socially patterned confounding by socio-economic position.

\section{1-5.4 FAMILIAL AGGREGATION IN NUTRIENT INTAKE PATTERNS: COMPARING INTERGENERATIONAL AND PRENATAL-POSTNATAL EFFECTS IN LIFEWAYS CROSS GENERATION COHORT STUDY}

doi:10.1136/jech.2011.142976a.39

${ }^{1,2}$ A Shrivastava, ${ }^{* 1,2} \mathrm{C}$ Murrin, ${ }^{1,2} \mathrm{C}$ Kelleher, for the Lifeways Cross Generation Cohort Study Steering Group. ${ }^{1}$ Health Research Board Centre for Diet and Health Research, University College Dublin, Dublin, Ireland; ${ }^{2}$ School of Public Health, Physiotherapy and Population Science, University College Dublin, Dublin, Ireland

Introduction Familial influence on dietary behaviours of children is expressed through genetic predispositions, maternal-environment and home-environment. Disaggregating such effects requires relatively rare cross-generational study designs. This analysis examined associations of maternal, paternal and grandparental dietary intake prenatally; and maternal and maternal-grandmothers dietary intake post-natally with child's dietary intake.

Methods Pre-natal dietary information was available for expectant mothers $(\mathrm{N}=1119)$, fathers $(\mathrm{N}=331)$, maternal-grandmothers $(\mathrm{N}=285)$, maternal-grandfathers $(\mathrm{N}=163)$, paternal-grandmothers $(\mathrm{N}=163)$, and paternal-grandfathers $(\mathrm{N}=96)$ through a validated food frequency questionnaire (FFQ). At 6 year follow-up, when children averaged age 5 , dietary information was re-collected for mothers $(n=558)$ and some maternal-grandmothers $(N=53)$ using the same FFO. A child's FFQ version was used for children $(\mathrm{N}=567)$. Association for energy, macronutrients and fibre intake were compared using Pearson's interclass and intraclass correlations. Nutrients were log transformed and adjusted for energy intake in interclass correlations.

Results Though the correlations were weakly moderate $(r<0.35)$ in strength, a clear pattern emerged. Positive statistically significant correlations were found for energy, macronutrients and fibre intake within children's nuclear families. Correlations were stronger for maternal postnatal-child pairs compared to maternal prenatal-child pairs. The father-child associations were significant (except for fat) but weaker than mother-child associations. Maternal grandmother-mother associations were found for protein, fat and fibre intakes. A significant positive intraclass correlations was observed in nutrient intakes of maternal grandmothers-mothers-child triads, not found in paternal lines.

Conclusion The stronger maternal associations with children's nutrient intake and existence of maternal grandmother-motherchild associations support evidence for maternal-environment programming influences. 